\title{
AN EXPERIMENTAL STUDY OF THE INITIATION AND PROGRESSION OF DAMAGE IN COMPRESSIVELY LOADED COMPOSITE LAMINATES IN THE PRESENCE OF A CIRCULAR CUTOUT
}

\author{
A. M Waas* \\ Dept. of Aerospace Engineering, Univ. of Michigan, Ann Arbor, MI. \\ C. D Babcock,jr., ${ }^{\dagger}$ \\ W. G Knauss ${ }^{\ddagger}$ \\ GALCIT, Pasadena, Ca. \\ January 16,1989
}

\begin{abstract}
A series of experiments have been performed to determine the mechanisms of failure in compressively loaded laminated plates in the presence of stress gradients generated by a circular cutout. Real time holographic interferometry and in-situ photomicrography of the hole surface, along with strain gage measurements are used to study the progression of failure.

The test specimens are multi-layered composite flat plates which are loaded in compression. The laminates are made up of two material systems, T300/BP907 and IM7/8551-7. Two different lay-ups of T300/BP907 and two different lay-ups of IM7/8551-7 are investigated.

The experimental results reveal that the failure is initiated as a localized instability in the $0^{\circ}$ plies at the hole surface approximately at right angles to the loading direction. This instability emanating at the hole edge and propagating into the interior of the specimen within the $0^{\circ}$ plies is found to be fiber microbuckling. Subsequent to the $0^{\circ}$ ply failure, extensive delamination cracking is observed with increasing load. The delaminted portions spread to the undamaged areas of the laminate by a combination of delamination buckling and growth, the buckling further enhancing the growth. When the delaminated area reaches a critical size, about $75-100 \%$ of the hole radius in extent, an accelerated growth rate

\footnotetext{
* Formerly, Graduate Research Assistant, GALCIT. Member AIA A

†Deceased. Formerly Professor of Aeronautics and Applied Mechanics.

¿Professor of Aeronautics and Applied Mechanics.

Copyright (C) American Institute of Aeronautics and Astronautics, Inc., 1989. All rights reserved.
}

of the delaminated portions is observed. The culmination of this last event is the complete loss of flexural stiffness of each of the delmainted portions leading to catastrophic failure of the plate.

\section{Introduction}

The use of Graphite/Epoxy laminates in aerospace engineering applications is abundant. These laminates offer an attractive combination of high strength and low density over their traditional metal counterparts. In many instances it is necessary to adopt techniques that are not conventional in characterising these materials, that differ from isotropic and homogeneous materials, in their response to mechanical load.

A considerable amount of research effort has been expended in attempting to understand the failure processes of composite laminates, especially in a compressive load environment. Such a task is made difficult by the several competing modes of failure that are prevalent in a given situation. From a design stand point the researcher needs to identify the most important aspect of the failure process. When structural components are made out of these laminates it is seldom that they will persist in an environment free of stress concentrations. Stress raisers can be lethal and are potential sites where damage can initiate in a structure. In the present work we shall be concerned with the failure processes in fibrous composite laminates in the presence of stress concentrations.

The open hole strengths of these laminates has been the subject of numerous investigations [1-4]. Figure 
1. depicts ultimate load carrying capacity of these laminates as a function of the normalised hole diameter. Mikulas [5], has shown that these results could be bounded by simple criteria based on notch sensitivity. If the material is notch insensitive, the failure strains are directly proportional to the reduction in cross-sectional area, while if the material is notch sensitive then it is postulated that the material fails when the strain concentration at the hole edge equals the failure strain of the material. It is seen that the latter assumption is too-conservative in that the failure strain is underestimated for $(\mathrm{a} / \mathrm{w})$ ratios less than 0.4 . This situation indicates the possibility that the strain concentration effect alone cannot possibly account for the failure process and that a localised failure mechanism may be present. Based on this premise Starnes et al. [1] carried out open hole strength testing of these laminates and reported a shear crippling type of failure to be prevalent in the vicinity of the hole prior to catastrophic failure. This conclusion was based on post-mortem examination of damaged specimen.

The progressive nature of failure, in many instances by various stages, is a characteristic property of laminated composites. Several different failure processes are prevalent when these laminates are stressed and what is required is to identify the key aspects of failure in a particular situation.

The present paper which is condensed from refs.[17], describes a detailed experimental investigation to understand the initiation and propagation of failure in laminates in the presence of a stress raiser. A companion paper [18] contains certain other aspects which are relevant to the present topic and thus, some repeatability is unavoidable.

The stress concentration effect is produced by choosing a flat plate with a centered circular hole and subjecting it to an inplane compressive load. The hole is carefully cut in order to insure that excessive local damage does not occur during the machining process. The damage initiation and propagation throughout the entire load history is studied via real time Holographic Interferometry and Photomicrography of the hole surface. Strain gage measurements are carried out on the IM7/8551-7 specimens. The results thus obtained are substantiated by post experiment examination of the damaged specimen Ultrasonically and under an Optical Microscope.

\subsection{Assembly}

\section{The Experiment}

Figure 2. is a photograph showing the arrangement of the various devices used in the experiment. On a pneumatically vibration isolated table, optical components are arranged to make a hologram and generate interferograms of the test specimen which is loaded by a table top compression device. An optical microscope is mounted on a tripod which is situated adjacent to the optical table by the testing machine. A $35 \mathrm{~mm}$ motor driven SLR camera attached to the microscope is used to take photographs of the test specimen hole surface, during the testing sequence. Recording and developing of the hologram is done on a thermoplastic plate which is housed in the body of the instant recording device(IRD) as marked in Figure 2. This device which is electronically controlled replaces the conventional wet chemical processes which are used had the recording being done on a photographic plate. A video camera placed behind the thermoplastic plate views the test specimen through the hologram. A VCR records thie interferometric pattern generated during the loading sequence. A TV monitor is used to view the interferograms during a typical test, which enables testing to be done interactively. In the following sections the main components of the experiment are described.

\subsection{Specimens}

The multi-layered laminated plates used in the tests were supplied by NASA-Langley Research Center. The composition of the laminae in the specimen consisted of Thornel-300 graphite fibers preimpregnated with American Cyanamide BP907 epoxy resin, and IM7 graphite fibers pre-impregnated in 8551-7 epoxy resin. The IM7 fibers have a smaller diameter $(5 \mu \mathrm{m})$ than the T300 fibers $(7 \mu \mathrm{m})$. The fiber volume fraction in both these systems is $0.5-0.6$. These laminates are typical of those used in the Aerospace Industry. Two types of lamina stacking sequence were used in the tests. The first designated Type $\mathrm{A}$ is 48 ply $[+45 /-45 / 0 / 0 /+45 /-$ $45 / 0 / 0 /+45 /-45 / 0 / 90]_{2 s}$ while the other, Type $B$ is obtained by rotating the first sequence by ninety degrees, the angles being measured with respect to the loading direction counterclockwise. Two sizes of specimen are used. The first designated " $\mathrm{S}$ " is $7.62 \mathrm{~cm} \times$ $10.2 \mathrm{~cm}$ and the other "L" is $12.7 \mathrm{~cm} \times 15.3 \mathrm{~cm}$.

\subsection{Specimen Supports}

The laminated plates need to be supported in a manner that allows a smooth load transfer from the testing machine on to the plates. To do this specimen holders made of mild steel are used in these tests. The diameters of the holders are chosen to be the widths of the specimen. The holders contain grooves of 1.27 $\mathrm{cm}$ depth and widths large enough to accomodate the specimen and the thin layers of bonding agent. The test specimen are inserted into the grooves and bonded to the support holders with DEVCON bonding agent. Edge stiffeners are attached to the sides of the speci- 
men to avoid plate buckling. These stiffeners increase the flexural stiffness of the plate but carry no load.

\subsection{Testing Machine}

The use of real time holographic interferometry requires vibration isolation of test specimen and testing device. As such, a special purpose testing device was designed and constructed for this purpose. Both tension and compression load can be applied using this device. At the commencement of a test one end of the specimen support holders is inserted into an adapter which threads onto the actuator arm of the loading device. A ball joint is attached to the other end. The mating part of the ball joint is positioned at the center of a $445 k N(100,000 l b f)$ load cell. The load cell is mounted on one of the flanged box beams of the testing machine. The load-time history is regulated through a servo controlled loading mechanism. The servo valve and associated accessories are mounted on a plate which fits on the cylinder of the loading device. A function generator is used to program the loading path.

\subsection{Holographic Interferometry}

Real time holographic interferometry is used in this study to record the out of plane displacement changes $\Delta w$ of the specimen during the loading sequence. The revelant details of the technique as applicable to the present experiment is given in section 2.7 . One novelty in the present experiment is the use of an electronically controlled process in the recording and development of a hologram. In conventional holography a wet chemical process is used in the development of the photographic glass plates. The time consumed during this process which is of the order of 7-9 minutes, is a significant portion of the total duration of a test which is of the order of 2 hours. During a continuous loading sequence, the information on the displacement history is lost during this development time. The electronic process refered to above and explained in the next section overcomes this drawback.

\subsection{The Instant Recording Device}

In the reconstruction process of a hologram the conventional Silver Halide photographic plates cause diffraction via an amplitude grating which is representative of the spatial distribution of light( amplitude and phase) that is emitted from the diffusely reflective surface of the object. However, the same task could be accomplished with a Phase grating i.e., a surface contour variation which is representative of the light off of the object. This is the basic idea behind the success of the thermoplastic recording device. The device has several stages of operation as detailed in [16].

\subsection{Experimental Procedure}

A schematic of the holographic interferometer used in this study is shown in Figure 3. It is a standard off axis holographic system. Proper choice of reference to object beam intensity ratio is important for producing the best holograms. With the use of the instant recording device, the quality of the hologram is insensitive to the exposure time. In the real time mode, the test specimen is illuminated in a state $S_{1}$ (say) with coherent light transmitted from the laser via the object wave path (this consists of the beam splitter, a beam expander-spatial filter, collimating lens). The corresponding load is $P_{1}$ (refer to Figure 4). A hologram is then recorded by exposing the thermoplastic plate positioned at the junction of the reflected light and reference beam. Upon completion of the first exposure the specimen which is continuosly loaded is viewed through a camera attached to a VHS recording system (a VCR and a monitor). The resulting image consists of fringes which are dynamical in nature representing contour maps of the changes, with respect to the state $\mathrm{S}_{1}$, of the out of plane displacement component $w$ of the stressed specimen. These fringes, together with the load cell reading are recorded. The load cell reading is obtained by focussing the display of the voltmeter, exhibiting the load cell reading, at the position of the circular hole in the plane of the test specimen. With increasing load on the specimen the fringe density becomes high, so that the resolution of the interferogram becomes poor. At this stage a new hologram of the test specimen is made and the above cycle of events is repeated. Thus, at the end of each experiment we obtain an interferometric recording of the complete load-out of plane displacement history of the specimen. This sequence of events is depicted in Figure 3(b).

Each of the holographic interference fringe field is related to the change in surface displacement of the specimen under load, by the vector expression

$$
n \lambda=\tilde{k} \cdot \tilde{d}
$$

where,

$n$-fringe number

$\lambda$-wavelength of the coherent laser light

$\tilde{k}$-sensitivity vector $\left(\tilde{i}_{2}-\tilde{i}_{1}\right)$

$\tilde{d}$-surface displacement vector at point of observation

$\tilde{i}_{1}$ and $\tilde{i}_{2}$ are the unit vectors in the illumination and observation directions respectively. In the present experimental setup the diffusely reflective surface of the specimen is oriented normal to the bisector of the angle $2 \alpha$, between $\tilde{i}_{1}$ and $\tilde{i}_{2}$ (Figure 3 ). Thus, since the sensitivity vector $\tilde{k}$, also points along this bisector, the 
interferometer senses only the out of plane displacement component $w$. Equation (1) then becomes

$$
\begin{aligned}
n \lambda & =2 w \cos \alpha \\
w & =\frac{n \lambda}{2 \cos \alpha}
\end{aligned}
$$

In the present experimental setup, the surface normal is $10^{\circ}$ off the observation direction. With $\lambda=0.633 \mu \mathrm{m}$, this shows that each fringe represents an out of plane displacement change of $0.321 \mu \mathrm{m}$.

During the entire loading program, the surface of the hole is viewed through a microscope at an oblique angle and photographs are taken at various times. Due to the oblique viewing angle, the hole surface appears curved on the photo-micrographs. A fibre optic cable attached to a high intensity lamp is used to illuminate the hole surface. The interferograms displayed on the TV monitor helps taking these photomicrographs interactively in an efficient manner.

The interferometric data reveal the changes in the $w$ displacement component. These changes may be the manifestations of internal damage. Thus, some specimen were loaded to a post initiation level but were unloaded prior to catastrophic failure. The unloaded specimen were sectioned in the region of stress concentration and were examined under an optical microscope. In the following section the results obtained from Interferometry, Photomicrography and Interior damage studies are presented.

\section{Results and Discussion}

Tables 1 and 2 summarise the test conditions and the results obtained. In these tables, "AS" for example, refers to type " $A$ ", small. $P_{i}$ is the load at which failure initiates, as detected by the inteferometer. $\mathrm{P}_{f}$ is the failure load of the specimen. The interferometric data are presented only in the immediate neighbourhood of the hole. The photomicrographs are only captured on one side of the hole surface.. The horizontal shiny stripes appearing on the photomicrographs are $0^{0}$ plies while vertical lines are pencil marks for purposes of reference.

Consider now the different stages of failure as exhibited by the interferometric results and substantiated by the photomicrographs. In the pre-damaged state an increase in the load on the specimen results in a uniform pattern of fringes. These fringes are a few in number and correspond to the thickness changes due to the poisson's ratio effect, but coupled with some rigid body motion and/or slight bending. The rigid body motion and the bending cannot be totally avoided in a compression test. The slight bending may be the result of any initial imperfection in the specimen. Added to this one must realise the high sensitivity of the measurement technique which is a function of the wave length of the laser light. (displacement changes as small as $0.321 \mu \mathrm{m}$ can be detected). In the discussion to follow, the T300/BP907 material system will be refered to as 'TB', while the IM7/8551-7 will be refered to as 'IM'.

Figure 5 shows a series of interferometric patterns of a type B specimen which correspond to the first instance at which internal damage was detected. In these interferograms, the axis of loading is horizontal. The numbers below each picture represent the load in $\mathrm{KN}\left(\left(4.448 \times 10^{3}\right)^{-1} l b f\right)$ corresponding to each frame, while $\mathrm{P}_{h}$ indicates the load at which the hologram was made for that particular set of frames(Reference load). Notice the highly localised fringe cluster adjacent to the hole edge at approximately $90^{\circ}$ to the loading direction. When any form of internal damage occurs that manifests itself as a localised perturbation in the normal displacement component $w$, the resulting interferometric patterns display this event as shown in this figure. A view of the hole surface corresponding to this fringe pattern is shown in frame 2 of Figure 6 . Notice the damage to the midplane $0^{0}$ plies which appear as a black spot. Adjacent to this spot is a delamination crack. Inspection of the photomicrographs prior to this event revealed no damage to the hole surface. In all experiments conducted it was revealed that always a perturbation in the Interferometric data corresponded to $0^{0}$ ply damage at the hole surface. This interferometric pattern is interpreted as a localised bulging of the surface just above the damage site. This localised damage to the $0^{0}$ plies is found to be the triggering of extensive delamination cracking and localised buckling of the delaminated portions, that follows subsequently. The above sequence of events is consistently observed in all of the Type B specimen. At this stage it is pointed out that the word damage is used to describe any form of destruction to the specimen caused by the application of mechanical load as picked up on the interferograms. The interferograms corresponding to the initiation event reveal the displacements within the damage zone to be increasing with increasing load. This is clear by noting a sharp increase in the fringe density within the damage zone as loading proceeds. Subsequent to the initiation, the remaining $0^{\circ}$ plies undergo failure while delaminations are seen to appear between various ply interfaces. An inspection of the sequence of photomicrographs in Figure $5 \mathrm{a}$ shows this. The delaminations undergo a stage of slow growth as shown in the next set of interferometric patterns (Figure 7). Here the reference state corresponds to a load $\mathrm{P}_{h}=159 \mathrm{kN}$ while the three frames span a load increase, $\Delta \mathrm{P}$ of $1.73 \mathrm{kN}$. The displacement patterns extracted from the interferograms in Figure 7, are shown 
in Figure 8. Here the displacements are measured with respect to displacements in areas remote from the hole edge. The origin of the axes is placed at the edge of the hole as indicated. The $\mathrm{X}$ and $\mathrm{Y}$ axes are scaled while the vertical $\mathrm{Z}$-axes representing $\Delta w$ is $1: 1$. The fringe patterns in Figure 7, reveal the displacements to be growing faster within the damage zone than the rest of the specimen. Further, the damage area is also seen to grow indicating that the delaminated portions are spreading outward approximately at right angles to the direction of applied load. The corresponding photomicrographs (Figure 6.) show the extent to which delamination cracking occurs. Notice the zero fiber breaks as well as the large (on the order of a ply thickness) mode I type displacements. These observations lead us to conclude that Delamination Buckling is the mechanism chiefly responsible for spreading of the damage to the undamaged areas of the plate. Once the delaminated portions reach a sizeable extent an increase in the growth rate is noticed. This is shown in the last series of interferograms (Figure 9). In these the fringe density within the damage zone is very high, so much so, that the resolution of the interferogram within this area is poor. However the manner in which these delaminations grow and respond can be deduced by follwing the fringe development within the damage area. It must be pointed out that playback of the recorded video information displays a continuos transition in the fringe data which enables following the damage progression un-interrupted as opposed to the limited number of frames displayed here. The present discussion is influenced from such a knowledge. The culmination of the last growth event is the complete loss of flexural stiffness of each of the delaminated portions, leading to catastrophic failure. These findings and conclusions are drawn from the consistency with which these failure events occur in all Type B specimen.

In a type $\mathrm{A}$ specimen ( $42 \% 0^{\circ}$ plies as contrasted with $8 \%$ in type $\mathrm{B}$ ), the mechanisms leading to failure initiation and propagation is as discussed before for type B specimen, apart from the levels of applied load at which the different events take place. This is evident from the results presented in Tables 1 and 2 . A sharp localised cluster of fringes is seen to form at the edge of the hole which spreads very rapidly with a small increase in applied load. The area in which the fringes are unresolvable extends out to about $80 \%$ of the hole radius, from the hole edge. A corresponding set of photomicrographs, typical of these specimen, is shown in Figure 10. Here, the initial $0^{\circ}$ fiber failure occurs at $139 \mathrm{kN}$. With increasing load the remaining $0^{0}$ plies undergo failure. Adjacent to some of the damaged $0^{0}$ plies, delamination cracking is found to occur. Notice the damage to all $0^{\circ}$ plies and the formation of surface buckles on both surfaces of the specimen. A picture of the damaged, unloaded specimen is shown in the last frame of Figure 10.

Bearing in mind the information obtained from the surfaces of the specimen, we now turn to a discussion of post experiment sectioning studies. Shown in Figure 11 is a typical C-scan map of a damaged specimen. In this figure, the extent of the damaged area is contained within about one hole radius. The damaged area is a function of the maximum load to which the specimen is loaded. Sectioning through the damaged area and observation under an optical microscope revealed several inclined narrow zones of fiber breaks within the $0^{0}$ layers, identified as Fiber Kink bands. A typical section through the damged area is shown in Figure 12. Here, the interior $0^{0}$ plies show several kinking failures. A typical complimentary kink band is shown in Figure 12(b). The geometry of a typical kink band is summarised in Figure 13. We note in Fig. 12(b) a kink band inclination $(\alpha)$ of $65^{\circ}$ to the load carrying direction. The lengths of the fibers in the inclined zone measure to 50-84 $\mu \mathrm{m}$. More importantly it must be pointed out that the region between the inclined zone appeared undamaged and oriented parrallel to the fiber direction. In a study by Evans et al.[10], carbon fiber bundles of width $\approx 400 \mu \mathrm{m}$ oriented in three orthogonal directions were set in a Graphite matrix and subjected to localised impaçt. Post-experimental examination revealed typical kink orientation angles $\alpha$ of $45^{\circ}-60^{\circ}$ and kink boundary orientation $\beta$ obeying $\beta \approx \frac{\alpha}{2}$ for $\operatorname{broad}\left(\frac{l}{d} \geq 0.1\right)$ kinks. Here $\beta$ is measured from the perpendicular to the fiber direction. For narrow kinks $\beta$ was found to increase and $\alpha$ random. Corresponding results in our study which fall into the category $\frac{l}{d} \geq 0.1$ indicate $\beta \approx 25^{\circ}-30^{\circ}$ and $\alpha \approx 15^{\circ}-30^{\circ}$. However, no definite trend between $\alpha$ and $\beta$ can be established. This type of kinking failure has also been observed in other studies dealing with compressive strengths of laminates (see for example [9]-[11]).

\subsection{Strain Gage Results}

It was desirable to obtain the local value of strain at which microbuckling occurred. To do so, some IM series specimen were mounted with standard foil strain gages. The strain gages were connected to a Wheatstone bridge in a single active gage configuration, and at each location back to back gages were placed to obtain both the in-plane axial strain in the direction of applied load as well as the bending strain. The gage outputs from the Wheatstone bridge were sent through an amplification stage and fed into a in-house computer for manipulation. Strain-load plots typical of type A and $B$ specimens are shown in Figure 14. In this figure, the response of the strain gages, near the hole edge 
( location $\mathrm{H}$ ), is shown. Notice that the strains are seen to increase linearly with increasing far-field load. When the far-field load reaches $\mathrm{P}_{\boldsymbol{i}}$ (corresponding to microbuckling of the $0^{0}$ plies), the load vs strain behavior shows a kink (marked 'A'), implying a localized softening. For the type B specimen ( Fig. 14(a)), with failure of other $0^{\circ}$ plies, the softening continues (marked ' $\mathrm{B}$ '), until reaching ' $\mathrm{C}$ ', when strain reversal is detected on one gage. This reversal occurs because the delaminated portions closest to the surface on which the gage is attached undergo buckling. The buckling results in tensile strains on the outer surface, due to bending, causing a net reduction in the compressive strain. For the type A specimen, beyond failure initiation, data can be collected only upto the point where surface buckles form due to delamination. At this time, the large out of plane displacements encountered during the rapid propagation and growth of the delaminated portions result in the strain gages being peeled off of the surface, disrupting the data aquisition. The averaged microbuckling strain for the two types of specimen are listed in Table 3.

\subsection{Summary of Common Features of Failure for Types $A$ and $B$.}

In this section we summarise the results presented so far and attempt to identify features that are common to types $\mathrm{A}$ and $\mathrm{B}$ laminates.

In both, types $A$ and $B$, the failure is initiated by a $0^{0}$ fiber failure at the hole edge approximately at right angles to the loading direction. In type A, with increasing load more $0^{\circ}$ fiber failure is seen to occur with simultaneous surface delamination buckling. These events occur very close to the catastrophic failure load. In type $B$, subsequent to $0^{0}$ fiber failure which occurs at around $75 \%$ of the ultimate failure load, gradual development of delamination cracking is observed, with the outermost delaminated sections undergoing buckling. In both types, the delaminations are seen to grow with increasing load. Delamination Buckling is found to be the mechanism responsible for the spreading of the damage. Finally, an accelerated growth of the delaminations is observed. At this time, the specimen fails catastrophically.

The mechanics of the $0^{0}$ fiber failure, which shows up at the hole edge and captured by the interferometer is found to be a localised microbuckling/fiber kinking. Information regarding fiber kinking published elsewhere and in here, have been obtained once the damage has taken place and the structure loaded into a postdamaged state. Qualitative information regarding the pre damaged state, from the interior of the structure, is difficult to obtain as these systems are opaque.

\section{Concluding Remarks}

In an attempt to understand the mechanisms for the initiation and spreading of damage in compressively loaded composite laminates containing a circular cutout, a detailed experimental investigation has been carried out. The investigation was aimed at recognizing the distinction between an unfailed laminate and a failed laminate and providing the transitional information that would serve to develop analysis capabilities with an underlying physical basis. This information is seldom obtained by following a purely theoretical path, and it was necessary that this problem be looked at experimentally. It is found that;

i The initiation of failure in all the laminate types investigated is by fiber microbuckling in the $0^{\circ}$ plies. This failure gradually develops at the hole surface in the form of a fiber jutout failure. When the far-field load reaches a critical value, the corresponding localized value of strain at the hole edge reaches a critical value of $\approx 8500 \mu$ strain (deduced from the IM series specimen). At this value of local strain, fiber microbuckling failure within the $0^{0}$ plies is seen to occur, resulting in a propagation of the damage into the interior of the specimen. The damage zone extends out to approximately $75 \%$ of the hole radius. The microbuckling process results in the $0^{\circ}$ fibers undergoing large rotations in-plane $(\mathrm{XY})$ as well as out-of-plane (XZ). This leads to the formation of narrow $\left(\frac{l}{d} \approx 7-11\right)$ bands of broken fiber segments that align themselves at an inclination to the loading direction. The initiation occurs at a far-field applied load which is $75-95 \%$ of the ultimate failure load of the specimen, the exact value depending on the laminate type. Thus, for all practical purposes the laminate can be termed "failed" once microbuckling of the $0^{0}$ plies occurs.

ii The surface manifestation of the initiation process is captured by the interferometer as a well defined perturbation in an otherwise smooth fringe pattern.

iii The interferometric data is substantiated by ultrasonic C-scanning, hole surface photomicrography and sectioning studies under an optical microscope.

iv Subsequent to $0^{\circ} \mathrm{ply}$ damage, delamination cracks are seen to form at ply interfaces close to the location of the $0^{0}$ plies as shown by the photomicrographs. The extent of the initial delaminated portion, the far field load at which the delaminaticn forms and the thickness of the delaminated portion, govern the occurrence of delamination buckling. 
v The buckling enhances growth of the delminated portions which spread to the undamaged areas of the laminate approximately at right angles to the direction of applied load.

vi When the delaminated area reaches a critical size, the growth process is seen to accelerate rapidly. The culmination of this last event is the catastrophic failure of the plate due to a complete loss of flexural stiffness of each of the delaminated portions. The load carrying capacity beyond initiation, at which stage delamination buckling/growth occurs, is dictated by the overall stiffness of the laminates. This, in turn, is governed by the $0^{0} \mathrm{ply}$ percentage. A mechanical model for the initiation of failure is presented elsewhere [19].

\section{Acknowledgments}

This research was supported by the National Aeronautics and Space Administration under Grant NSG-1483. The authors are appreciative of this support. The interest and encouragement of Dr. James H. Starnes, Jr., NASA langley, in pursuing this work is acknowledged.

\section{References}

1. Starnes, J. and Williams, J.G. (1982), "Failure characteristics of $\mathrm{Gr} /$ Epoxy struc tural components loaded in compression", NASA TM 84552.

2. Rhodes, M., Mikulas, M. and McGowan, P. (1982), "Effect of orthotropic properties and panel width on the compression strength of $\mathrm{Gr} /$ Epoxy laminates with holes", AIAA paper No. 82-0749.

3. Shuart M.J and Williams J.G. (1986), "Compression behaviour of $[+45 /-45]$ domin- ated laminates with a circular hole or impact damage", AIAA journal, vol. 24, No. 1.

4. Knauss J., Starnes J. and Henneke E. (1978), "The compressive failure of $\mathrm{Gr} /$ Epoxy plates with circular holes", NASA CR 157115, N78-24295.

5. Mikulas M. (1980), "Failure prediction techniques for compression loaded compo- site laminates", NASA CP 2142.

6. Babcock C.D. and Waas A.M. (1985), "Effect of stress concentrations in composite structures", GALCIT SM Report 85-12.

7. Rosen B.W. (1985), "Mechanics of composite strengthening", Fiber composite materials, Am.soc. for Metals seminar.
8. Schuerch H. (1966), "Prediction of compressive strength in uni-axial Boron fiber -metal matrix composite materials", AIAA journal, vol. 4, pp102-106.

9. Chaplin C.R. (1977), "Compressive fracture in uni-directional glass reinforced plastics", $J$. of Mat.Science, vol. 12, pp347-352.

10. Evans A. and Adler A. (1977), "Kinking as a mode of structural degradation in Carbon fiber composites",Acta Metallurgica, vol. 26, pp725-738.

11. Hahn H. and Williams J.G. (1984), "Compression failure mechanisms in unidirectional composites", NASA TM 85834.

12. Budiansky B. (1983), "Micromechanics", Computers 8 Structures, vol. 16, No.1-4, pp3-12.

13. Chai H. (1982), "Growth of impact damage in compressively loaded laminates", P.hD thesis, Caltech, Pasadena, Ca.

14. Vest C.M. (1979), Holographic Interferometry , Wiley

15. Pfaff R.D. (1986), private communication, Caltech, Pasadena, Ca.

16. Owners Manual-HC300,301 (1986), Newport Research Corporation, Ca., USA.

17. Waas A. (1988), "Compression failure of fibrous laminated composites in the presence of stress gradients: experiment and analysis", P.hD thesis, Caltech, Pasadena, Ca.

18. Waas A., Babcock C.D and Knauss W.G (1989), "An experimental study of compression failure of fibrous laminated composites in the presence of stress gradients", Int. J. of solids and structures, special commemorative issue.

19. Waas A., Babcock C.D and Knauss W.G (1989), "A mechanical model for elastic fiber microbuckling", proc. ASME Energy Technology Conference and Exhibition, Houston, TX. 
Table 1 Experimental results for T300/BP907 laminates.

\begin{tabular}{|c|c|c|c|c|}
\hline SPEC./TYPE & $\begin{array}{l}\text { HOLE } \\
\text { DIA. } \\
(\mathrm{cm})\end{array}$ & $\begin{array}{c}\text { THICKNESS } \\
(\mathrm{mm})\end{array}$ & $P_{i}(K N)$ & $\mathrm{P}_{f}(\mathrm{KN})$ \\
\hline $\mathrm{TB} 1-\lambda \mathrm{S}$ & 2.54 & 7.51 & 126.0 & $127.0^{\circ}$ \\
\hline TB2-AS & 2.54 & 7.51 & 124.0 & $125.0^{\circ}$ \\
\hline TB10-AS & 2.54 & 7.53 & 124.0 & 127.0 \\
\hline TB12. $\overline{S S}$ & 2.54 & 7.53 & 125.0 & 127.0 \\
\hline TB7-BS & 2.54 & 7.51 & 64.0 & 91.0 \\
\hline TB13-BS & 2.54 & 7.47 & 69.0 & 90.0 \\
\hline TB6-BL & 2.54 & 7.51 & 127.0 & 168.0 \\
\hline TB11-BL & 2.54 & 7.53 & 123.0 & $165.0^{\circ *}$ \\
\hline TB9- $\bar{B} \bar{L}$ & 2.54 & 7.51 & 119.0 & 169.0 \\
\hline TB14-AS & 1.90 & 7.47 & 130.0 & 142.0 \\
\hline TB17-AS & 1.90 & 7.53 & 128.0 & $143.0^{* *}$ \\
\hline TB16-BS & 1.90 & 7.47 & 70.0 & $97.0^{\circ *}$ \\
\hline TB18-BS & 1.90 & 7.53 & 79.0 & 110.0 \\
\hline TB15-AS & 1.27 & 7.47 & 152.0 & $161.0^{\circ *}$ \\
\hline
\end{tabular}

"Load held constant atter initiation.

"Maxinum load sustained. Specimen unloaded for microscopic examination.

Table 2 Experimental results for IM7/8551-7 laminates.

\begin{tabular}{|l|l|c|c|c|}
\hline SPEC./TYPE & $\begin{array}{l}\text { HOLE } \\
\text { DIA. } \\
(\mathrm{cm})\end{array}$ & $\begin{array}{c}\text { THICKNESS } \\
(\mathrm{mm})\end{array}$ & $P_{i}(\mathrm{KN})$ & $\mathrm{P}_{f}(\mathrm{KN})$ \\
\hline \hline IM2-AS & 2.54 & 7.44 & 128.5 & 144.1 \\
\hline IM4-AS & 2.54 & 7.31 & 135.7 & 141.9 \\
\hline IM5-AS & 2.54 & 7.33 & 124.8 & 129.0 \\
\hline IM7-AS & 2.54 & 7.33 & 125.0 & 133.2 \\
\hline IM16-AS & 2.54 & 7.32 & 119.2 & 128.3 \\
\hline IM20-AS & 2.54 & 7.36 & 127.7 & 132.8 \\
\hline IM21-AS & 2.54 & 7.32 & 125.0 & 134.3 \\
\hline IM29-AS & 2.54 & 7.32 & 125.9 & 133.2 \\
\hline IM1-BS & 2.54 & 7.38 & 68.7 & 97.8 \\
\hline IM3-BS & 2.54 & 7.32 & 72.0 & 88.3 \\
\hline IM6-BS & 2.54 & 7.36 & 70.5 & 92.1 \\
\hline IM8-BS & 2.54 & 7.36 & 67.2 & 89.4 \\
\hline IM9-BS $^{4}$ & 2.54 & 7.33 & 72.1 & 89.8 \\
\hline IM19-BS & 2.54 & 7.36 & 68.9 & 76.3 \\
\hline IM32-BS $^{4}$ & 2.54 & 7.30 & 60.0 & 90.3 \\
\hline IM24-BL & 2.54 & 7.36 & 127.0 & 136.3 \\
\hline IM25-BL & 2.54 & 7.44 & 123.2 & 165.5 \\
\hline IM28-BL & 2.54 & 7.33 & 114.3 & 164.1 \\
\hline
\end{tabular}

${ }^{4}$ Maximum load sustained. Specimen unloaded for microscopic examination.

Table 3 Averaged local strain values at failure initiation-IM series specimens.

\begin{tabular}{|c|c|}
\hline SPEC./TYPE & $\begin{array}{c}\text { AVG. STRAIN } \\
\text { AT INITIATION } \\
(\mu \text { strain })\end{array}$ \\
\hline $\mathrm{A}$ & 8385 \\
\hline $\mathrm{B}$ & 8612 \\
\hline
\end{tabular}

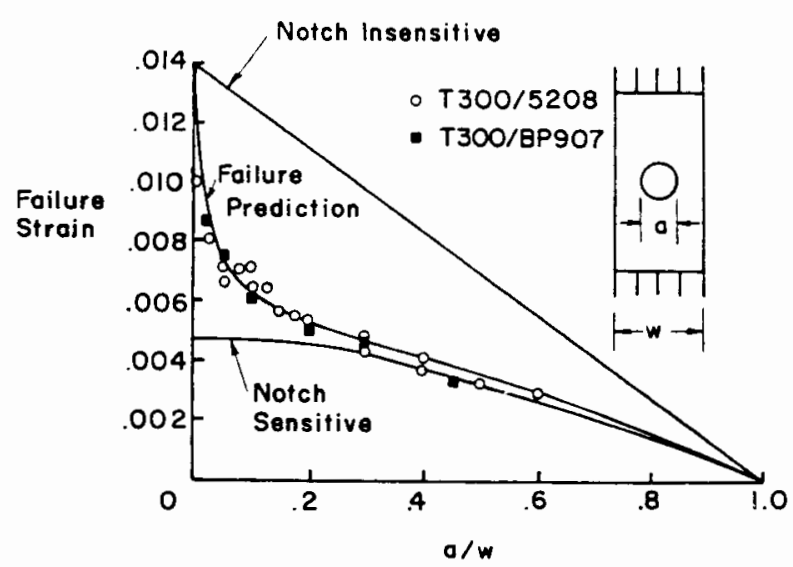

Figure 1 Summary of experimental results for T300/BP907 and T300/5208 composite laminates. $w=13 \mathrm{~cm}$ (from ref. [1]).

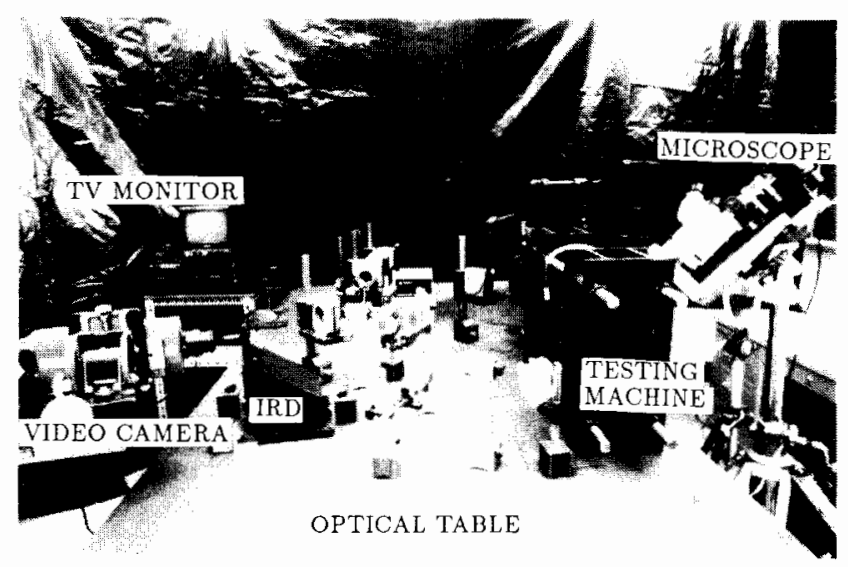

Figure 2 Experimental set up.

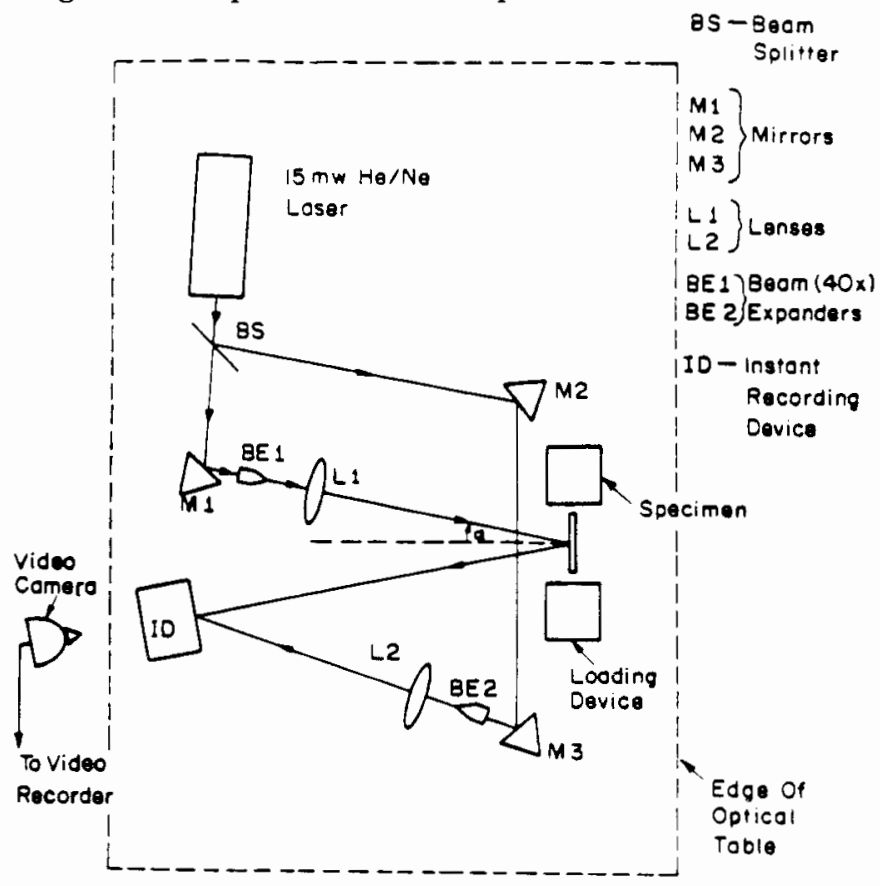

Figure 3 Schematic of holographic interferometer. 


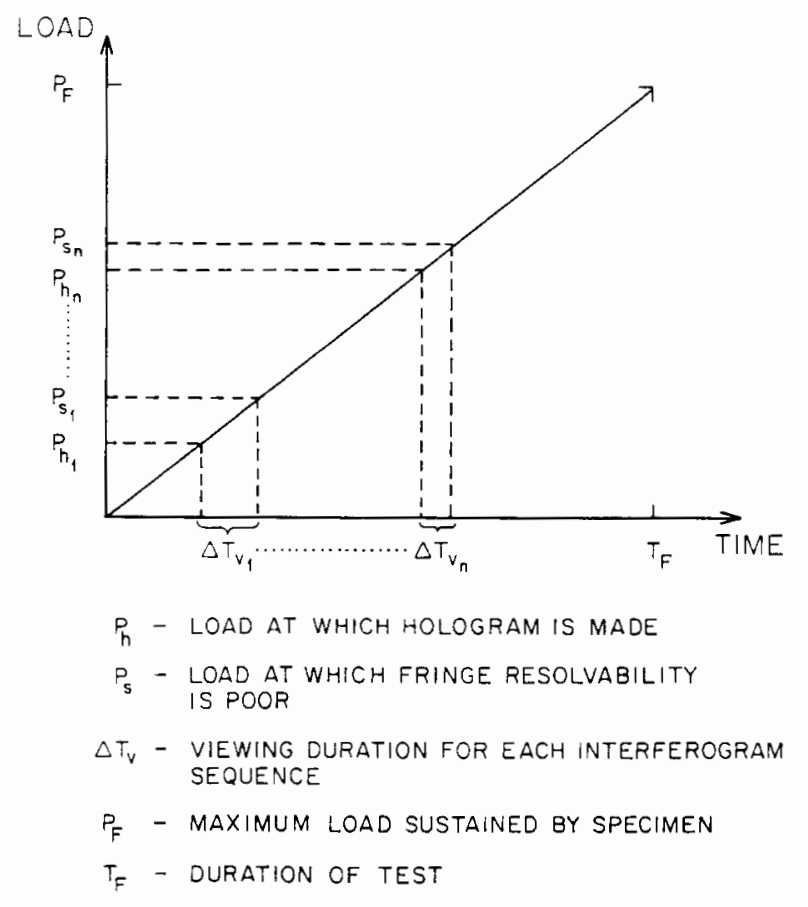

Figure 4 Experimental procedure for generating interferograms.

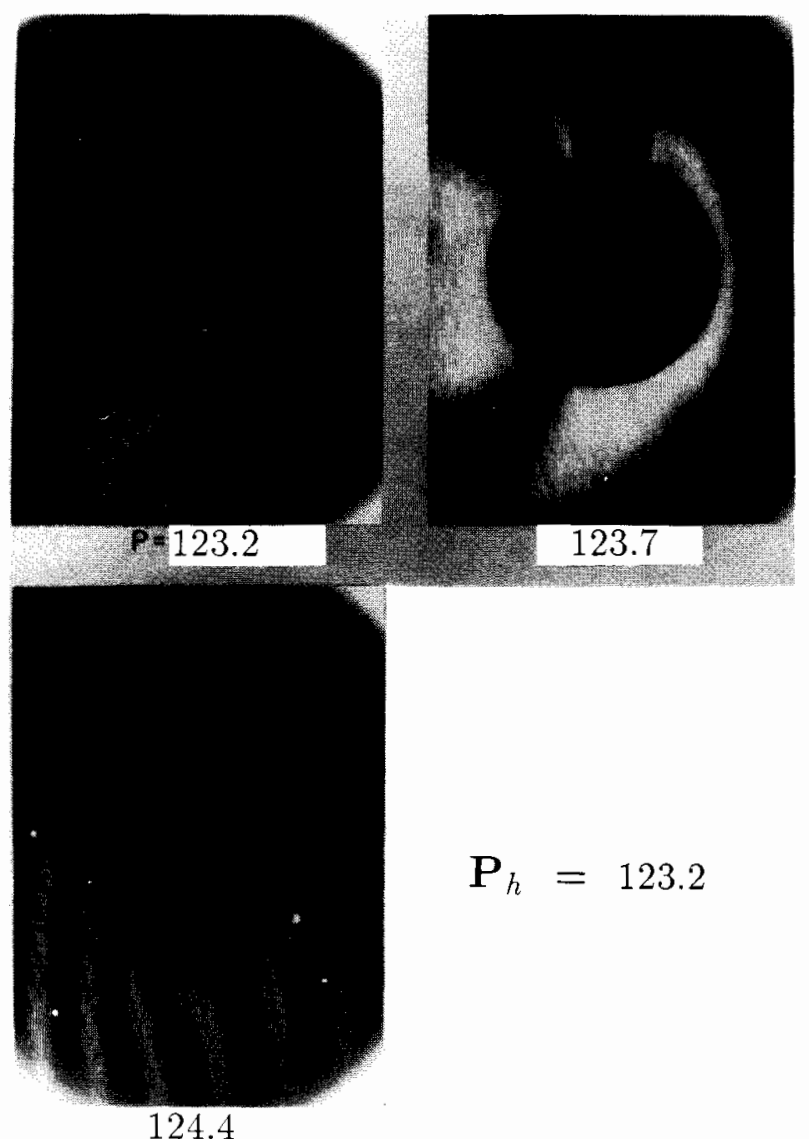

Figure 5 Initiation of damage ( spec. TB11).

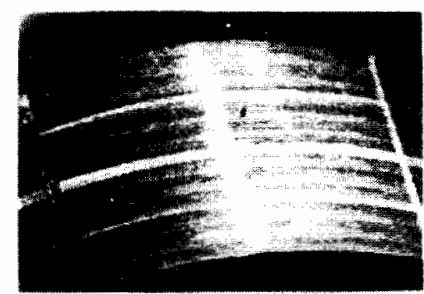

$P=66.7$

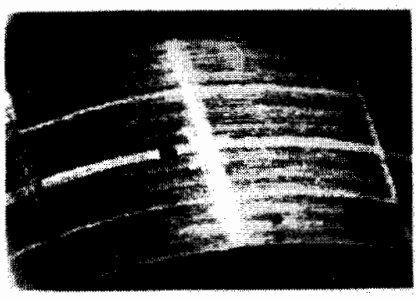

123.2

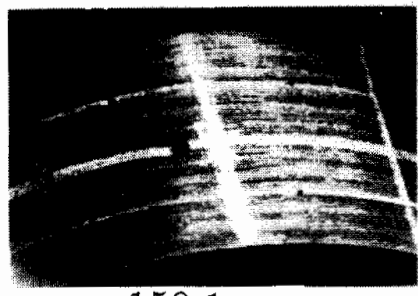

159.1

Figure 6 Hole surface photomicrographs of specimen TB11 ( Type B).
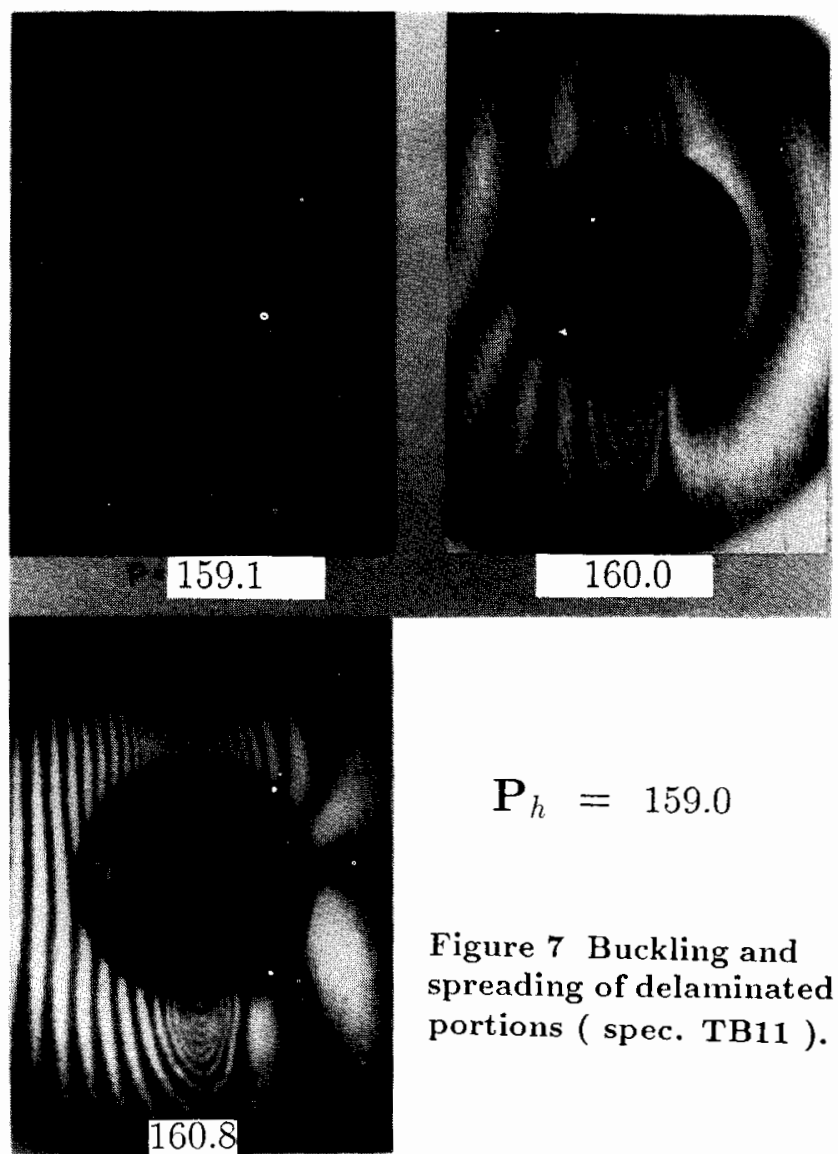

$$
\mathbf{P}_{h}=159.0
$$

Figure 7 Buckling and spreading of delaminated portions ( spec. TB11). 


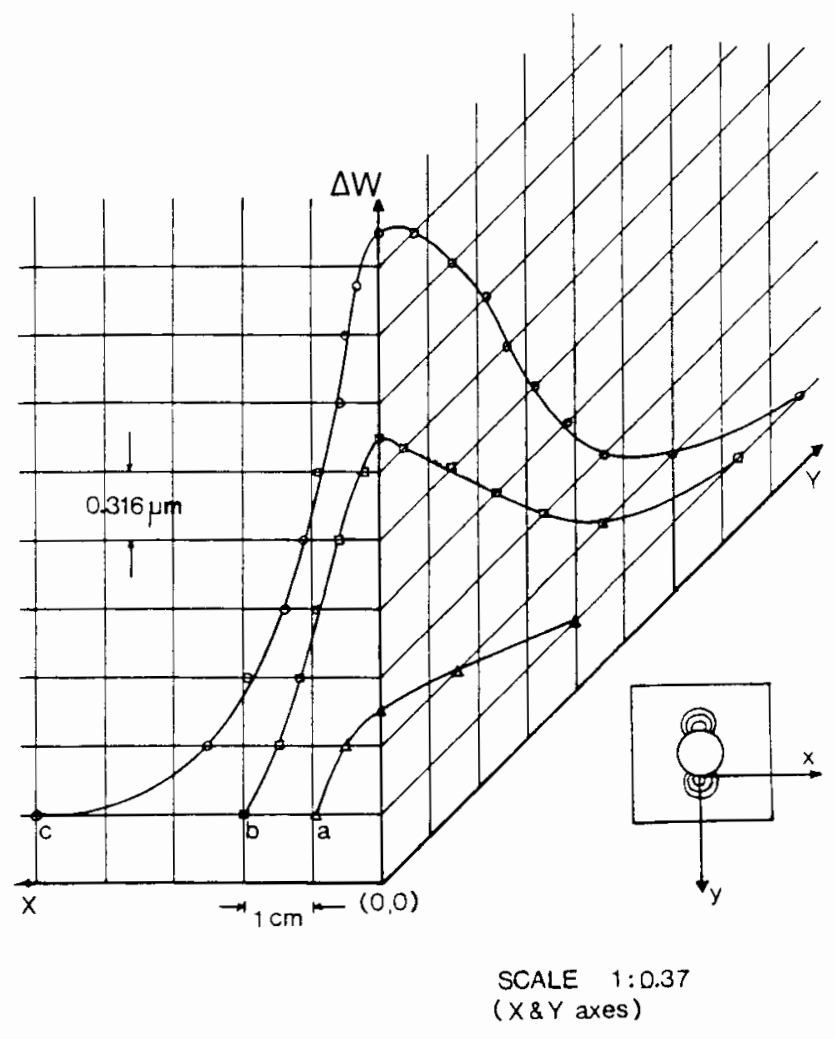

Figure 8 Summary of displacements corresponding to Figure 7.

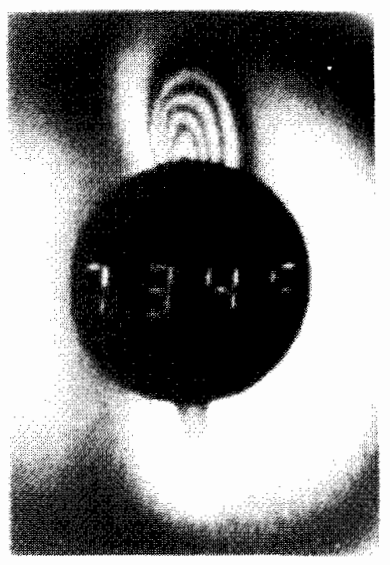

163.4

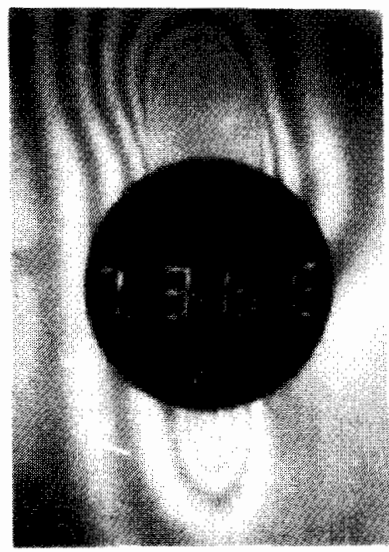

163.9

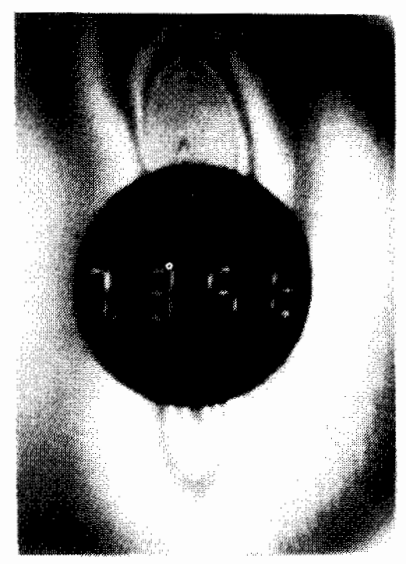

163.6
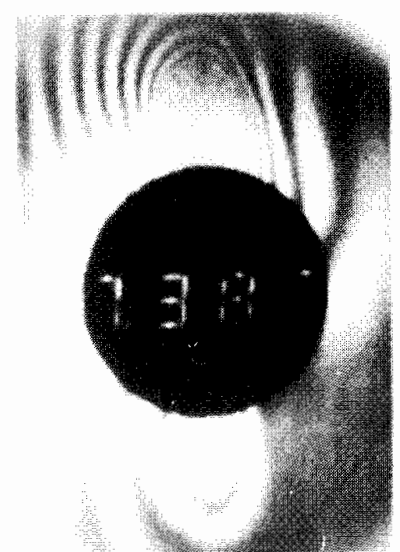

164.3

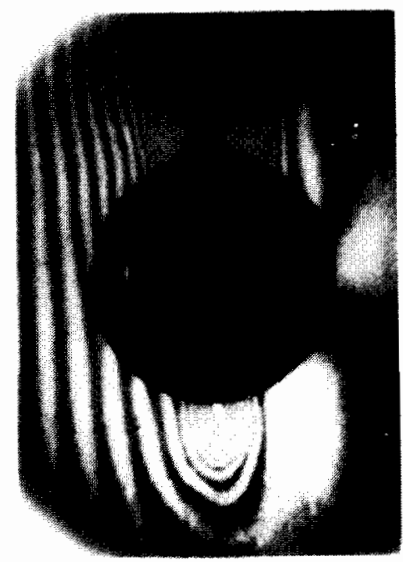

164.5

$$
\mathbf{P}_{h}=163.3
$$

Figure 9 Buckling and spreading of delaminated portions just prior to catastrophic failure (specimen TB11).

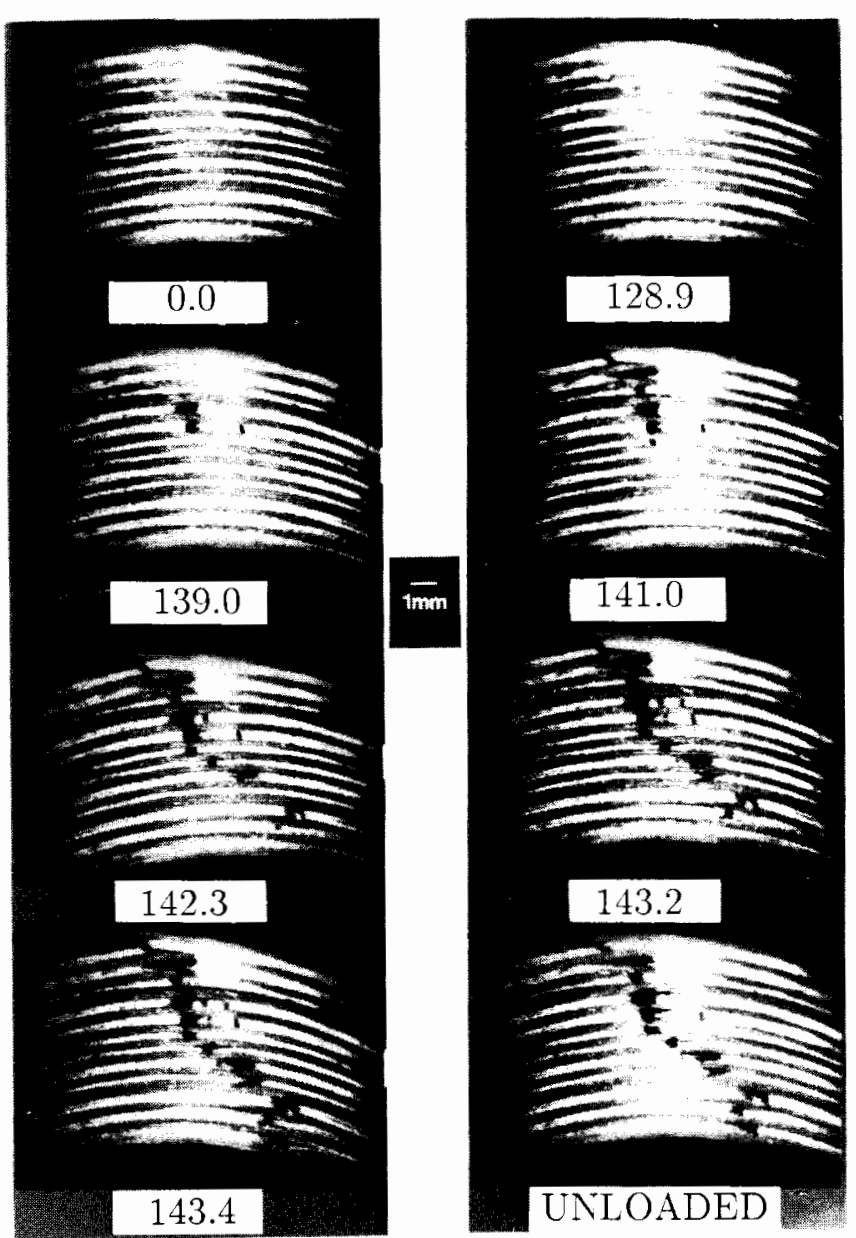

Figure 10 Hole surface photomicrographs showing damage progression ( spec. TB17). 


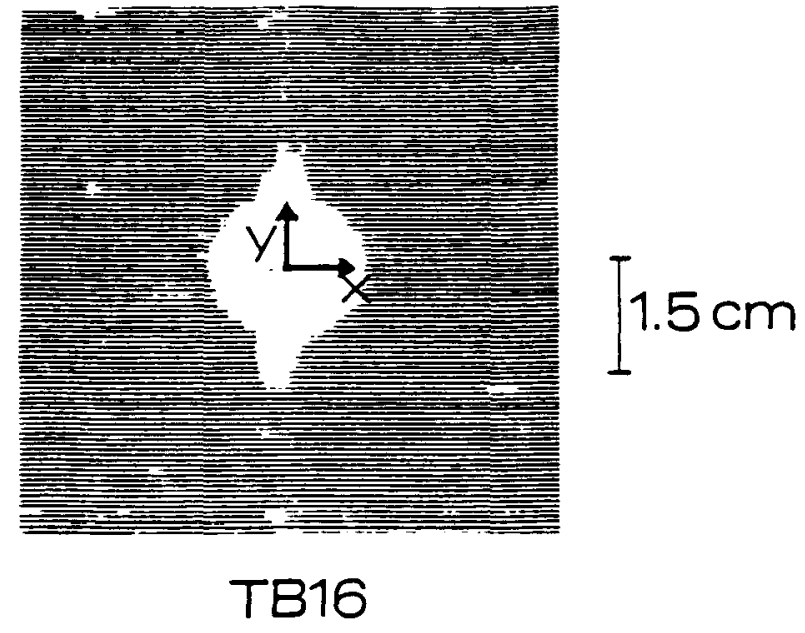

Figure 11 C-scan map of typical damaged specimen (spec. TB16).

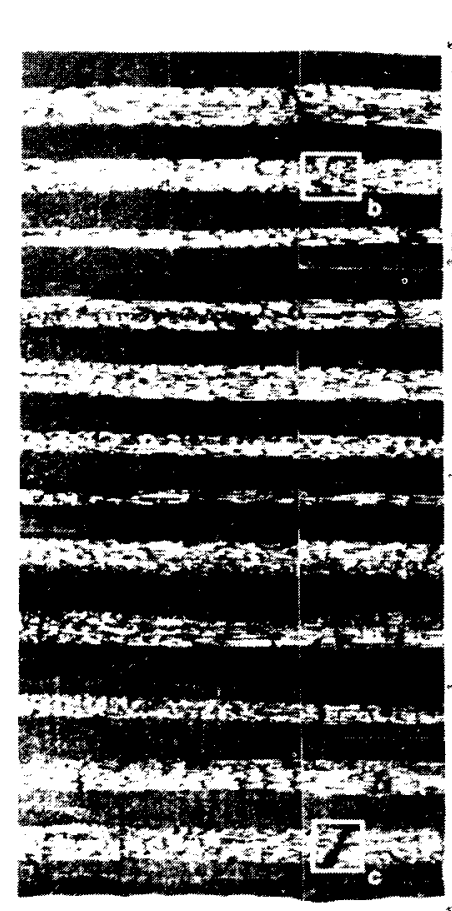

$126 \mathrm{~mm}$

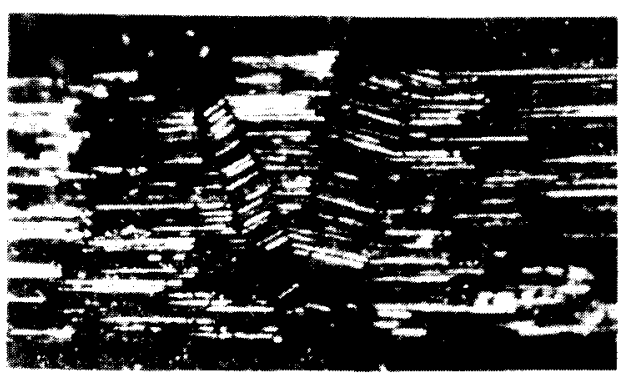

b

$16 \mathrm{~mm}$

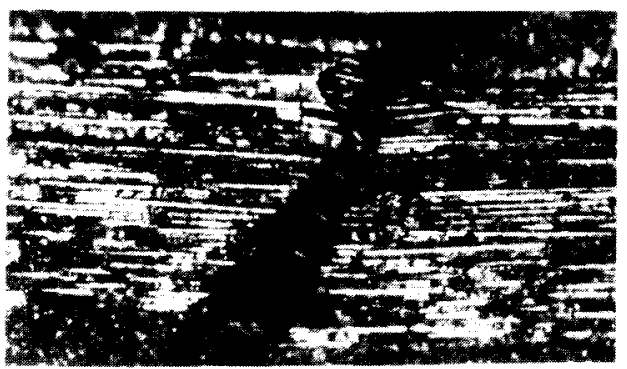

Figure 12 Internal damage at the vicinity of the hole ( spec. TB15 ). a) Entire cross section. b) Complimentary kink in second $0^{0}$ layer from top. c) Magnified view of damage to bottom $0^{0}$ layer.
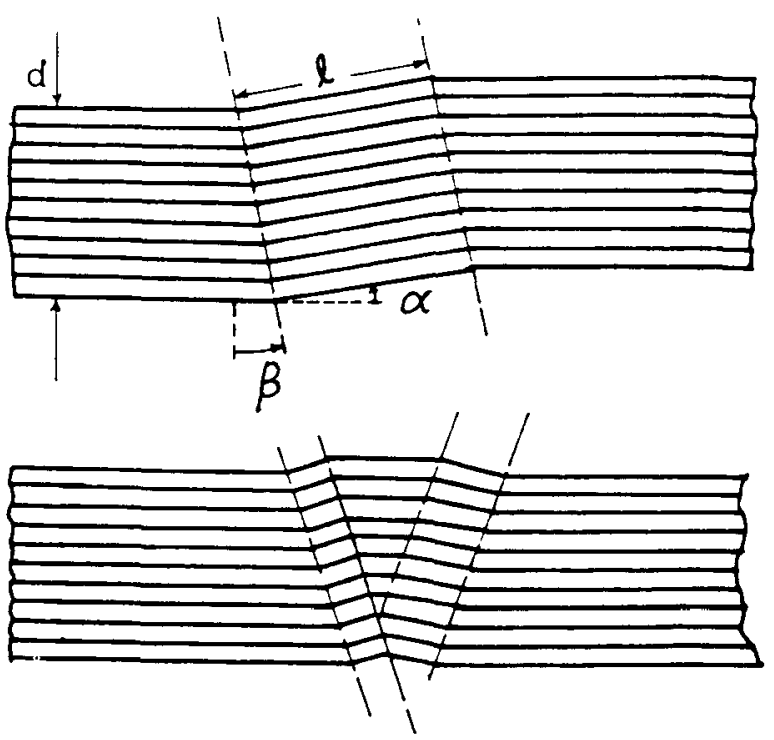

W

Figure $12 \ldots \ldots$

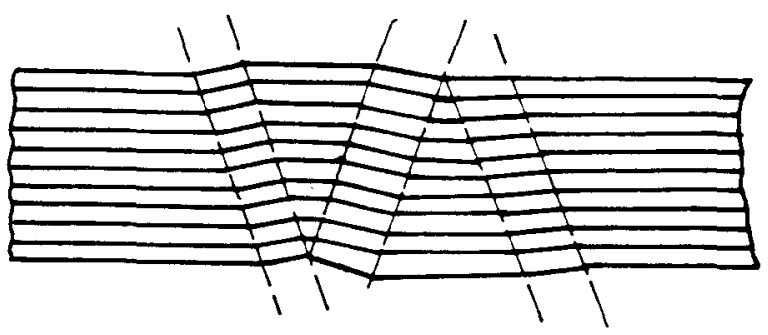

Figure 13 Geometry of typical kink bands. 

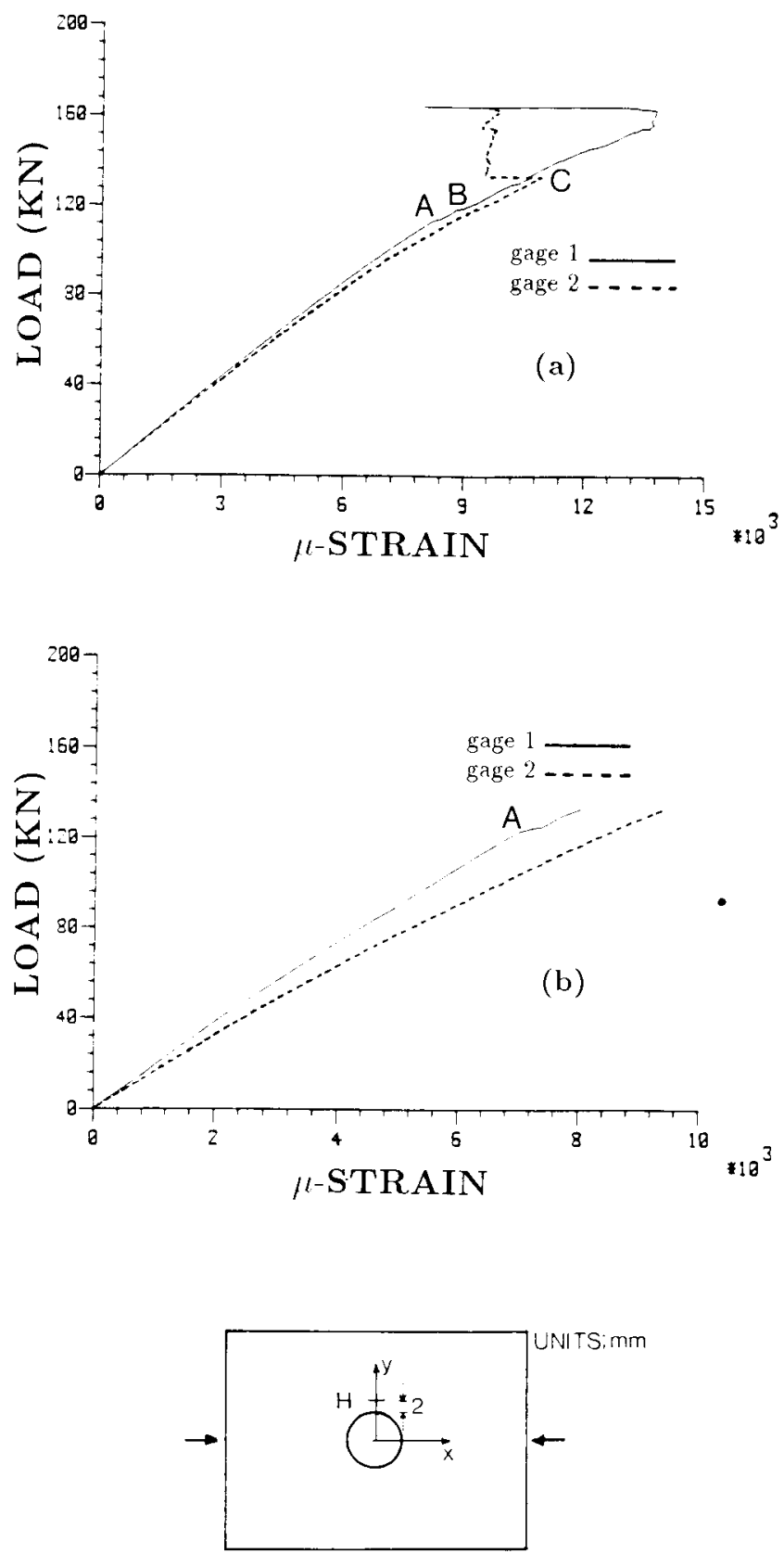

Figure 14 Strain gage response for (a) type B ( spec. IM28) and (b) type A ( spec. IM21) specimens. 